\title{
Active Comparator Drug
}

National Cancer Institute

\section{Source}

National Cancer Institute. Active Comparator Drug. NCI Thesaurus. Code C68612.

A therapeutically active agent that is intended to provide baseline measurements for the experimental protocol of a clinical trial. 